\title{
Investigating the Effect of Multidimensional Review Text and Anonymity on Review Helpfulness: An Empirical Investigation in the Context of Employer Review Sites
}

\author{
Pubali Mukherjee \\ Binghamton University, SUNY \\ pmukher1@binghamton.edu
}

\author{
Srikanth Parameswaran \\ Binghamton University, SUNY \\ sparames@,binghamton.edu
}

\author{
Rohit Valecha \\ University of Texas at San Antonio \\ rvalecha6446@gmail.com
}

\begin{abstract}
Employer review sites have grown popular over the last few years, with 86 percent of job seekers referring to reviews in these sites before applying to job positions. Though review helpfulness is studied in various contexts, it has received limited attention in the employee review context. In an attempt to solicit unbiased reviews, these sites allow an option of keeping reviewer information anonymous. Besides, these sites provide review text in multiple dimensions. We investigate review helpfulness focusing on the anonymity of the reviewers, and the role of review text in multiple dimensions. We use a publicly available Glassdoor dataset to model review helpfulness using a Tobit regression. The results show that anonymity and review length in multiple dimensions of review text positively impact review helpfulness. Moreover, anonymity positively moderates the review length in the cons section.
\end{abstract}

\section{Introduction}

With the growing popularity of digital media, online word of mouth has become pervasive across various sectors. Be it online shopping [1], dining [26], searching for jobs, downloading mobile applications [2], and selecting movies [3], consumers look for Electronic Word of Mouth (eWOM) through online reviews. Prior literature has shown that eWOM influences several decisions of consumers, including attitude formation, improving sales [4], generating revenue, and building brand loyalty [5]. Given the impact of online reviews on the consumer decisionmaking process, research has focused on investigating review helpfulness because not all reviews are equally valuable and perceived as helpful by consumers. There is a plethora of factors that determine the helpfulness of online reviews [6]. Though, prior research evaluates perceived helpfulness for online reviews of products and services across domains ranging from electronics, books [7], music, toys, video games, movies [3], hotels, restaurants [8] and travel tours [9], yet, understanding the role of various factors predicting the helpfulness of online employer reviews from the perspective of a job seeker is scarce.

Unlike restaurant or movie reviews, job seekers use employer reviews to make career decisions that can have life-changing consequences. Moreover, there is a more extensive evaluation period (in the order of years) before a reviewer (i.e., employee) rates the reviewed entity (i.e., the employer). Whereas, in other contexts (barring electronics like mobile phones) such as restaurants, tours, movies, the reviews are written based on one-off casual encounters. Recent studies in Glassdoor have found that around 86 percent of job seekers go through these reviews before applying [10]. This makes it important to investigate what makes an online review helpful from a job seeker's perspective. Prior research on employer review sites has focused on mining and unraveling job attributes mentioned in an employer review $[11,12]$. However, the antecedents of review helpfulness have received less attention.

In the context of online employer review sites, we highlight two gaps in prior research on review helpfulness. First, some job review portals like Glassdoor use multiple sections in the review text for pros and cons (commonly referred to as multidimensional review text), unlike Yelp that utilizes only a single section for the review text. Multiple sections could make it easier for review readers to delineate the positive and the negative information about the reviewed entity. Prior research focuses on multidimensional star rating systems and its consequences on user-generated content [14]. However, there is a lack of research on the role of multiple dimensions of review text on review helpfulness. 
Second, unlike the offline context where employees may be hesitant to engage in critical word of mouth, reviewers in the online context can provide critical feedback about their employers by being anonymous [13]. Employees can choose to be anonymous, mainly when posting a critical online review in fear of being reprimanded by their company. So, anonymity plays a more crucial role in the employer review context. Prior research shows that anonymity negatively influences review helpfulness. However, we lack an understanding of the role of anonymity and its influence on review helpfulness in employer review sites.

Along with this backdrop, we address the following research questions in the context of employer review sites:

(i) What is the impact of review text in multiple dimensions on review helpfulness?

(ii) How does reviewer anonymity affect the helpfulness of reviews?

We address these research questions using a publicly available Glassdoor dataset and modeling review helpfulness in the job context. We investigate review text in two dimensions (pros and cons), and reviewer anonymity on review helpfulness. We also examine the moderating role of reviewer anonymity in the relationship between multidimensional review text on review helpfulness.

Our study makes two theoretical contributions to the field of online review helpfulness. One, we extend prior research by theorizing that review text in multiple dimensions impacts review helpfulness. However, prior research looks at the impact of review text in a single dimension [18]. Two, while prior research on the effect of anonymity on review helpfulness has shown mixed (and predominantly negative) findings [6], we show that in the context of employer reviews, anonymity signals review credibility and positively impacts review helpfulness. Besides, we show that review anonymity has a moderating effect on the impact of review text in multiple dimensions on review helpfulness. Our research has important practical implications for online review sites' design and crafting guidelines and policies for employees writing reviews.

The remainder of the paper is organized as follows: The next section provides the literature review on review helpfulness in the employer review context. The literature review section is followed by our research model, where we elaborate on the multidimensional reviews, reviewer anonymity, and moderating impact of anonymity on multidimensional review text. This section is followed by research methodology, where we introduce the sample, variables, model specification, and results. Subsequently, we present the discussion section to discuss our key findings, theoretical, and managerial implications. Finally, we conclude the paper with conclusions, limitations, and future work.

\section{Literature Review}

In this section, we discuss the concepts of review helpfulness in the context of employer reviews. We look at prior research on the antecedents of helpfulness, the importance of review texts in multiple dimensions, and the critical role of reviewer anonymity in the employer review context.

\subsection{Review Helpfulness}

Review helpfulness represents the extent to which a consumer perceives that a review influences their decision-making process [18]. There are different filtering procedures and voting systems used to capture a review's perceived helpfulness [2]. The websites hosting online reviews use a straightforward question "Was this review helpful?" Review readers can mark their answer with a "Yes" button or an "Upvote." These responses are used to measure the helpfulness of an online review.

Prior research has focused on the antecedents of helpfulness pertaining four aspects: (1) review related factors: length [19], consistency [20], text emotions [21], posting time [21], readability [37], (2) reviewer related factors: information disclosure, reviewer picture, demographics [22], reviewer reputation to be an expert and trustworthy [20], (3) review reader related factors: psychological factors like intrinsic features of the review like the argumentative feature driving the decision making of the reader [23] and how well the reader can identify himself/herself with the review or the reviewer identification measure [24], and (4) environment related factors: how and where the reviews are being posted, the context of the review, to what extent the review is visible to the readers [25], the influence of voting system on the reviews [27], the medium through which the reviews are sought [28]. However, research in the employer review context is scanty.

\subsection{Employer Reviews}

In the employer reviews area, studies have focused on the job attributes and employer attributes mentioned in a review [29]. For example, there are studies on brand attributes of the employer [12], and corporate value (innovation, integrity, teamwork) using text mining of Glassdoor reviews [30]. Some studies have examined 
the effect of eWOM on employee intentions and job seeker's attitudes [29].

Some employer reviews are designed in such a way that they display review text in multiple dimensions. For example, Glassdoor reviews are separated into pros and cons sections. When reviewers write their reviews, the review submission form provides two separate input fields that prompt them to write about an employer's positive and negative aspects. The text written in these fields is shown under different sections named pros and cons when presented to a review reader. Thereby, these reviewing systems provide a structure for the review writer and the review reader. Multidimensional review text makes it easier to read and comprehend a review for a reader. In the employer review context, these sections delineate the organization's pros and cons. It facilitates informed decision making for job seekers. However, there is a lack of research investigating the impact of multidimensional review text on review helpfulness.

Besides, reviewer anonymity plays a critical role in employer reviews. Review writers may like to maintain anonymity while writing reviews because they do not want to face negative consequences from the employers [13]. Owing to this, employer review platforms like Glassdoor, provide the option of leaving the fields of job title blank so both current and former employees can provide their feedback without disclosing their job titles. Moreover, review readers expect reviews to be anonymous, unlike in other review platforms like Amazon or Yelp [13]. Despite this, anonymity and its effect on review helpfulness in the context of employer reviews has received little attention.

This paper investigates factors influencing helpfulness in an employer review portal, specifically multidimensional review text and reviewer anonymity, to address the gaps mentioned above. We conceptualize multidimensional review text using the number of words in the review's pros and cons sections [15]. Furthermore, we conceptualize anonymous reviewers as those that do not disclose their job titles i.e., conceal part of their identities while writing reviews about the employer.

\section{Research Model}

In this section, we discuss the research model focusing on the role of review text length in the pros and cons sections of the employer review sites, the anonymity of the reviewers, and the moderating impact of anonymity on multiple dimensions of review text and review helpfulness.

\subsection{Multidimensional Reviews}

In multidimensional reviews, longer reviews include details about the job and the organization. They provide an elaborate explanation about the specifics, which improve information diagnosticity [18].

Job seekers usually look for a detailed overview of the organization to make informed decisions [18]. Detailed descriptions in the review texts' multiple dimensions like the pros section and the cons section of the review make the reviews complete and informative. Specifically, the more reviewers write for the organization's pros and cons in their reviews, the more information a job seeker gets about the company while reading the review. They get a comprehensive overview of what people like and what people dislike about the organization. Job seekers perceive such informative and complete reviews to be helpful $[6,18,31,32]$.

Job seekers look at the pros to know more about the organizations' growth and opportunities, which include perks, benefits, work culture, compensation, and worklife balance. The detailed information about each of them makes them confident to make decisions [18]. Similarly, review readers look for negative information (cons) of the organization, including corporate politics, a hindrance to the path to promotion, mismanagement, and corruption. Prior research shows a positive relationship between review length and review helpfulness [6]. So, we state the following hypotheses:

H1: Review length in pros section has a positive effect on the helpfulness of a review

H2: Review length in cons section has a positive effect on the helpfulness of a review

\subsection{Role Anonymity}

Prior studies have investigated the effect of reviewer anonymity on online review helpfulness and shown mixed findings [6]. Studies have shown a predominantly negative impact of reviewer anonymity on review helpfulness. Anonymous reviews are perceived to be less credible since the readers fail to trust an anonymous reviewer [16]. Studies have also revealed a nonsignificant impact of anonymity on review helpfulness [33, 17].

In employer reviews, review writers are apprehensive of giving feedback, specifically critical feedback. They are scared about the consequences they might face from their employers if they get identified [13]. They are mostly hesitant to disclose themselves and prefer remaining anonymous while providing job reviews. 
Reviews by role anonymous reviewers express their personal experiences and feelings openly [33]. Readers attribute privacy and freedom in review writing for reviews written by anonymous reviewers [33]. Consequently, they perceive the reviews written by anonymous reviewers to be honest and genuine [6]. Therefore, we argue that the reviewer's anonymity will have a positive impact on helpfulness in the employer context. So, we hypothesize:

H3: Role anonymity has a positive effect on review helpfulness.

\subsection{Moderating Effect of Role Anonymity}

Besides the main effect, role anonymity also moderates the effect of multidimensional review length on review helpfulness.

When the reviewers write lengthy reviews in the pros section, they do not expect negative consequences. Instead, they might get benefits for writing positives about their employer. There are fewer reasons for a review writer to be anonymous and write lengthy text in the pros section. When review writers write lengthy text in the pros section and opt to be anonymous, it creates an information asymmetry for the readers - they become skeptical. For example, a software developer working in a small company writing a lot in the pros section is easily identified and can expect perks and benefits for writing good things about the company. Despite these benefits, if the review writers are not disclosing their roles, it creates an information asymmetry, which reduces the review's credibility in the eyes of the review reader. Therefore, we propose:

H4a: Role anonymity negatively moderates the effect of review length in pros section on review helpfulness

Role anonymity amplifies the positive effect of review length in the cons section on helpfulness. The more the reviewers write in the cons section, the more apprehensive they are of their employer's repercussions. So, anonymity is best suited for reviewers providing a critical evaluation of their employer (compared to a positive assessment) [33]. A lengthy review in the cons section written by an anonymous reviewer represents the most honest and credible form of critical employer evaluation for a review reader. Consequently, review readers would trust a lengthy review written by an anonymous reviewer thinking that a non-anonymous reviewer would not divulge the ground reality owing to the repercussions. Thus, we propose:
H4b: Role anonymity positively moderates the effect of review length in cons section on review helpfulness.

Apart from these independent variables, we control for additional variables. The review related factors that we use as control are the review emotional tone in the pros section and the cons section. Positive tone conveys a sense of pleasantness, while negative tone conveys disappointment [35]. Review rating is a measure of review valence, which can drive review helpfulness [35]. In accordance with prior research, both linear and quadratic review rating are used as control variables. [14]. Another antecedent of review helpfulness is review visibility [35]. Lastly, we are controlling for the employment status of the reviewer with the organization (i.e., whether they are current or former employees). Figure 1 illustrates our research model.

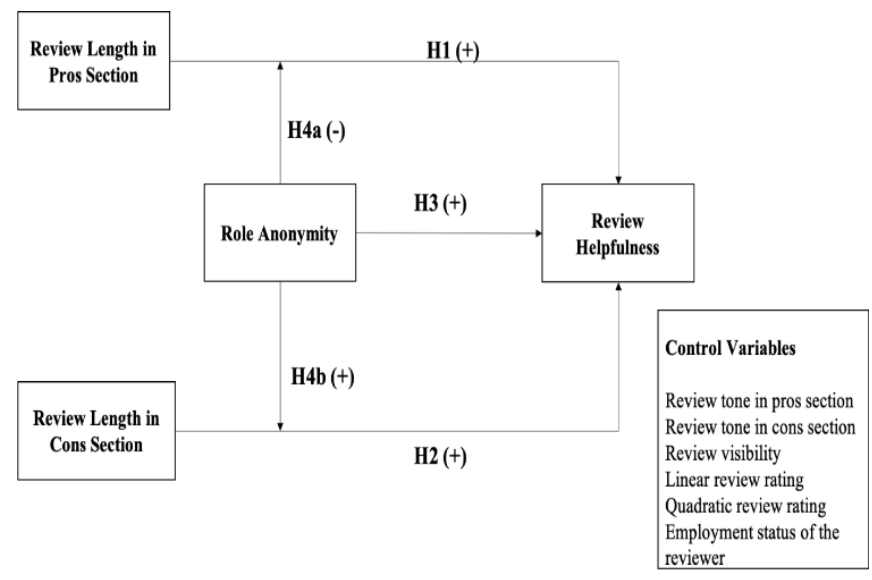

\section{Figure 1. Research Model of Review Helpfulness}

\section{Research Methodology}

\subsection{Sample and Data Collection}

We used a publicly available Glassdoor dataset containing online employer reviews from various worldwide from 2008 to 2019 [36]. Using a Python library, we removed non-English reviews. Also, we removed redundant reviews. We ended up with 66,480 online employer reviews, which were utilized for the data analysis. To compute the variables of interest, from this dataset, we utilized the review text, review star rating, review date, helpfulness votes received, and the reviewer information disclosed in the review. The review text had multiple dimensions in pros and cons. 
Everyone has free access to Glassdoor, read the reviews, and mark a review as helpful.

To calculate textual measures in pros and cons, we use automated text analysis within Linguistic Inquiry and Word Count (LIWC) [34]. LIWC uses a dictionarybased approach and maps different words of the text to the predefined psychological categories. LIWC was used to calculate word count and emotional tone in the pros and cons sections.

\subsection{Variables}

Dependent Variable. The dependent variable is review helpfulness. It was operationalized as the number of helpfulness votes a review receives.

Independent Variable. The independent variables are review length in the pros and cons sections and reviewer role anonymity. Review length was operationalized by calculating the number of words in each section [15] using LIWC. RLpros and RL cons denote it. Reviewer anonymity is an indicator variable operationalized by a dummy variable, roleanon coded 1 for an anonymous reviewer, and 0 when the role is provided.

Control Variable. Prior research has established that review sentiment significantly impacts review helpfulness [37]. We control for the emotional tone of the review text in the pros section and the cons section. The emotional tone measures are calculated automatically using the LIWC's proprietary emotional tone measure [2]. High values indicate a positive emotional tone, and low values indicate a negative emotional tone. They are denoted by Tonepros and Tone $e_{\text {cons }}$ in our model. We control for review rating, which denotes the star rating provided by the employee while writing the review. Prior research posits that review rating indicates a reviewer's extremity of attitude towards the reviewed entity and acts as a key variable impacting review helpfulness [35]. Prior studies have also shown that quadratic review rating significantly impacts helpfulness [18]. Both linear and quadratic review ratings are used as control variables. We added the time of the review as another control variable using a continuous variable measuring the year in which each review was written. Prior research posits elapsed time as an indicator of review visibility, with highly visible reviews gathering higher helpfulness votes [35]. Further, this control variable will account for any design-related changes in the review website. Lastly, we controlled for the employment status of the reviewer. It is an indicator variable measured as 1 for a current employee and 0 for a former employee. Review readers might ascribe more trust towards a review written by former employees who are more likely to be honest. The definitions of the variables are provided in Table 1.

\begin{tabular}{|c|c|}
\hline \multicolumn{2}{|c|}{ Table 1. Definition of the variables } \\
\hline Variables & Definition \\
\hline$R L_{\text {pros }}$ & Review length in pros section \\
\hline $\mathrm{RL}_{\text {cons }}$ & Review length in cons section \\
\hline Tonepros & Review tone in pros section \\
\hline Tone cons & Review tone in cons section \\
\hline Status & $\begin{array}{l}\text { Relationship of the reviewer with the } \\
\text { organization (Current vs. Former) }\end{array}$ \\
\hline roleanon & $\begin{array}{l}\text { Reviewer not disclosing their identity } \\
\text { (Anonymous vs. Non anonymous) }\end{array}$ \\
\hline elapsedtime & Year when the review was written \\
\hline Rating & Linear overall rating of the review \\
\hline Rating^2 & Quadratic overall rating of the review \\
\hline
\end{tabular}

\subsection{Model Specification}

To identify the hypothesized effects, we use the Tobit regression. We use Tobit regression because our dependent variable is censored in nature [18]. The variable helpfulness is bounded by the extremes. Job seekers may vote a review helpful if they indeed found value in a review. They cannot provide a more extreme value of helpfulness. Besides, even if they did not find the review helpful, they cannot downvote a review in Glassdoor (so zero is the lowest value of helpfulness although helpfulness could be negative). Thus, the resulting model for predicting helpfulness is:

$$
\begin{aligned}
& \text { Helpful }=\beta_{1}+\beta_{2} * R L_{\text {pros }}+\beta_{3} * R L_{\text {cons }}+\beta_{4} * \text { Tone }_{\text {pros }} \\
& +\beta_{5} * \text { Tone } \\
& \beta_{8} * \text { ens }+\beta_{6} * \text { Status }+\beta_{7} * \text { roleanon }+ \\
& \beta_{11} * \text { roleanon } * \text { R } L_{\text {pros }}+\beta_{12} * \text { Rating }+\beta_{10} *\left(\text { Rating }{ }^{\wedge} 2\right)+ \\
& \text { roleanon*RL } L_{\text {cons }}+€
\end{aligned}
$$

\subsection{Results}

\begin{tabular}{|l|l|l|l|l|}
\hline \multicolumn{5}{|c|}{ Table 2. Tobit regression result for review } \\
helpfulness \\
\hline & $\begin{array}{l}\text { Estim } \\
\text { ate }\end{array}$ & $\begin{array}{l}\text { Std. } \\
\text { Error }\end{array}$ & $\begin{array}{l}\text { Z } \\
\text { Value }\end{array}$ & $\operatorname{Pr}(>\mid \mathrm{zl})$ \\
\hline Constant & 0.10 & 0.44 & 22.37 & $<0.001$ \\
\hline RLpros & 0.033 & 0.00 & 33.67 & $<0.001$ \\
\hline RL $_{\text {cons }}$ & 0.024 & 0.00 & 33.21 & $<0.001$ \\
\hline Tone $_{\text {pros }}$ & 0.01 & 0.00 & 9.98 & $<0.001$ \\
\hline Tone cons & 0.00 & 0.00 & 4.72 & $<0.001$ \\
\hline Status & 0.86 & 0.08 & 10.76 & $<0.001$ \\
\hline Roleanon & 0.71 & 0.12 & 5.72 & $<0.001$ \\
\hline
\end{tabular}




\begin{tabular}{|l|l|l|l|l|}
\hline Elapsedtime & -0.50 & 0.02 & -22.30 & $<0.001$ \\
\hline Rating & -6.06 & 0.15 & -40.40 & $<0.001$ \\
\hline Rating^2 & 0.67 & 0.02 & 29.12 & $<0.001$ \\
\hline RL pros ${ }^{*}$ roleanon & $\begin{array}{l}0.007 \\
R_{\text {cons }}{ }^{\star} \text { roleanon }\end{array}$ & 0.00 & -0.32 & 0.748 \\
\hline
\end{tabular}

Table 2 presents the results of our estimation. Review length in pros has a positive significant effect on helpfulness $(B=0.033, p<0.001)$. Review length in cons also has a positive significant effect $(B=0.024$, $p$ $<0.001$ ) on helpfulness. Hence, hypotheses 1 and 2 are supported. Role anonymity has a positive significant effect on helpfulness $(\beta=0.710, \mathrm{p}<0.001)$. Therefore, Hypotheses 3 is supported. The moderating effect of role anonymity on review length in the pros section predicting helpfulness is not significant $(B=-0.007$, $p$ $>0.05$ ). Therefore, Hypotheses $4 \mathrm{a}$ is not supported. However, the moderating effect of role anonymity on review length in the cons section predicting helpfulness has a positive and significant association $(B=0.0031$, $p$ $<0.05)$ with review helpfulness. Therefore, hypothesis $4 \mathrm{~b}$ is supported. We find significant effects for our control variables.

\section{Discussion}

\subsection{Theoretical Implications}

Our study makes two theoretical contributions to the field of online review helpfulness. One, we extend prior research by theorizing that review text in multiple dimensions impacts review helpfulness [18]. In terms of review helpfulness, review readers expect elaborate information, both in pros and cons. Higher review length in the pros and cons section facilitates decision making for the job seekers, and they perceive the reviews as helpful. Review text in the pros and the cons section makes it easier for job seekers to delineate the positive and the negative information about an employer. Our study is the first to examine the impact of multiple dimensions of review text on review helpfulness. Prior research looks at the impact of review text in a single dimension [6]. Although prior literature looks at multidimensionality in reviews, the focus has been on the star ratings and the consequences on usergenerated context [14].

Two, by examining the positive effect of role anonymity, we extend the boundaries of review helpfulness literature by contextualizing it to the employer reviews. Prior research has predominantly shown a negative effect of anonymity on review helpfulness [6]. Studies have also demonstrated an insignificant relationship between anonymity and helpfulness $[33,19]$. In our study, we found that role anonymity positively impacts review helpfulness. In the employer context, review readers believe anonymous reviews are more credible, honest, and genuine.

We hypothesized and tested the moderating role of role anonymity in the relationships between review length in the pros and cons sections on review helpfulness. We found that role anonymity does not significantly moderate the effect of review length in the pros section on helpfulness. On the other hand, role anonymity amplifies the impact of review length in the cons section on helpfulness. The non-significant finding of the moderating role of role anonymity on the review length of the pros section implies that review readers do not worry about reviewer anonymity when learning about the employer's positives. However, this finding needs to be probed further.

\subsection{Managerial Implications}

Our research has important implications for practice. One, our positive effect of review length in the pros and cons section on helpfulness justifies the investment made by employer review sites in such advanced design features. Two, our findings imply that review sites should incentivize review writers to write lengthy text in the pros and the cons sections. Three, our results on role anonymity suggest that review writers who wish to be anonymous could rather give generic names to their roles while writing online reviews to get helpful votes. Four, our moderating effects imply that being anonymous while writing more content in pros will not affect the chances of receiving helpfulness votes. On the contrary, being anonymous while writing more content in the cons will increase their chances of receiving helpful votes.

\section{Conclusions}

\subsection{Limitations}

Our work has two limitations. First, we did not account for the number of views, a measure of review visibility that influences helpfulness [6]. This was a limitation of our data. However, we have used the elapsed time that could be a proxy for review visibility in our analysis. Second, our we did not incorporate company related variables like company rating, and company size in our model. So, caution must be exercised while generalizing these findings to all the companies. 


\subsection{Future Work}

We have analyzed the impact of role anonymity on review helpfulness in the employer review context. One possible direction is to analyze how role anonymity moderates other factors such as employment status. The second possible direction is examining the impact of the multiple dimensions of review star rating, such as perks, work-life balance, and benefits on review helpfulness.

\section{References}

[1] Agnihotri, Arpita, and Saurabh Bhattacharya, "Online review helpfulness: Role of qualitative factors", Psychology \& Marketing 33.11, 2016, 10061017.

[2] Cao, Qing, Wenjing Duan, and Qiwei Gan, "Exploring determinants of voting for the "helpfulness" of online user reviews: A text mining approach", Decision Support Systems 50.2, 2011, 511521.

[3] $\mathrm{Hu}, \mathrm{Nan}$, Ling Liu, and Jie Jennifer Zhang, "Do online reviews affect product sales? The role of reviewer characteristics and temporal effects", Information Technology and Management 9.3, 2008, 201-214.

[4] Archak, Nikolay, Anindya Ghose, and Panagiotis G. Ipeirotis, "Deriving the pricing power of product features by mining consumer reviews", Management science 57.8, 2011, 1485-1509.

[5] Srivastava, Vartika, and Arti D. Kalro, "Enhancing the helpfulness of online consumer reviews: the role of latent (content) factors", Journal of Interactive Marketing 48, 2019, 33-50.

[6] Rietsche, R., Frei, D., Stöckli, E., \& Söllner, M., Not all Reviews are Equal-a Literature Review on Online Review Helpfulness, in European Conference on Information Systems, Stockholm-Uppsala, Sweden, 2019: AIS.

[7] Hu, N., Bose, I., Koh, N. S., \& Liu, L., Manipulation of online reviews: An analysis of ratings, readability, and sentiments. Decision support systems, 52(3), 2012, 674-684.

[8] Wu, Philip Fei, "In search of negativity bias: An empirical study of perceived helpfulness of online reviews", Psychology \& Marketing 30.11, 2013, 971 984.

[9] Shin, S., Chung, N., Xiang, Z., \& Koo, C., Assessing the impact of textual content concreteness on helpfulness in online travel reviews. Journal of Travel Research, 58(4), 2019, 579-593.

[10] Glassdoor, 2019, "A Guide to the Ultimate Candidate Experience", available at: https://www.glassdoor.com/employers/blog/a-guide-tothe-ultimate-candidate-experience/.
[11] Luo, N., Zhou, Y., and Shon, J, "Employee Satisfaction and Corporate Performance: Mining Employee Reviews on Glassdoor. com", Thirty Seventh International Conference on Information Systems, Dublin 2016 (ICIS 2016), Association for Information Systems: Dublin, Ireland.

[12] Dabirian, A., Kietzmann, J., \& Diba, H., "A great place to work!? understanding crowdsourced employer branding”, Business Horizons, 2016, 60, 197-205

[13] BusinessGrow, "Don't Review Your Employer on Glassdoor Until You Read This!", 2017, available at: https://businessesgrow.com/2017/07/26/glassdoor/.

[14] Chen, Pei-Yu, Yili Hong, and Ying Liu, "The value of multidimensional rating systems: Evidence from a natural experiment and randomized experiments", Management Science 64.10, 2018, 46294647.

[15] Kong, D., Yang, J., Duan, H., \& Yang, S., Helpfulness and economic impact of multidimensional rating systems: Perspective of functional and hedonic characteristics. Journal of Consumer Behaviour, 19.1, 2020, 80-95.

[16] Lee, S. and Choeh, J.Y., "Exploring the determinants of and predicting the helpfulness of online user reviews using decision trees", Management Decision, 55.4, 2017, 681-700.

[17] Kwok, L. and Xie, K.L., "Factors contributing to the helpfulness of online hotel reviews: Does manager response play a role?", International Journal of Contemporary Hospitality Management, Vol. 28 No. 10, 2016, pp. 2156-2177.

[18] Mudambi, Susan M., and David Schuff, "Research note: What makes a helpful online review? A study of customer reviews on Amazon. com", MIS quarterly, 2010, 185-200.

[19] Zhou, Shasha, and Bin Guo, "The order effect on online review helpfulness: A social influence perspective", Decision Support Systems 93, 2017, 7787.

[20] Cheung, Cindy Man-Yee, Choon-Ling Sia, and Kevin KY Kuan, "Is this review believable? A study of factors affecting the credibility of online consumer reviews from an ELM perspective," Journal of the Association for Information Systems 13.8, 2012, 2.

[21] Fang, B., Ye, Q., Kucukusta, D., \& Law, R., Analysis of the perceived value of online tourism reviews: Influence of readability and reviewer characteristics. Tourism Management, 52, 2016, 498506.

[22] Gao, S., Tang, O., Wang, H., \& Yin, P., Identifying competitors through comparative relation mining of online reviews in the restaurant industry. International Journal of Hospitality Management, 71, 2018, 19-32.

[23] Liu, H., Gao, Y., Lv, P., Li, M., Geng, S., Li, M., \& Wang, H., Using argument-based features to predict 
and analyse review helpfulness, 2017, arXiv preprint arXiv:1707.07279.

[24] Davis, Joshua M., and Deepti Agrawal, "Understanding the role of interpersonal identification in online review evaluation: An information processing perspective", International Journal of Information Management 38.1, 2018, 140-149.

[25] Hu, Ya-Han, and Kuanchin Chen, "Predicting hotel review helpfulness: The impact of review visibility, and interaction between hotel stars and review ratings", International Journal of Information Management 36.6, 2016, 929-944.

[26] Huang, N., Burtch, G., Hong, Y., \& Polman, , .Effects of multiple psychological distances on construal and consumer evaluation: A field study of online reviews. Journal of Consumer Psychology, 26(4), 2016, 474-482.

[27] Kuan, K. K., Hui, K. L., Prasarnphanich, P., \& Lai, H. Y. What makes a review voted? An empirical investigation of review voting in online review systems. Journal of the Association for Information Systems, 2015, 16(1), 48-71.

[28] Askalidis, Georgios, Su Jung Kim, and Edward C. Malthouse, "Understanding and overcoming biases in online review systems", Decision Support Systems 97, 2017: 23-30.

[29] Carpentier, Marieke, and Greet Van Hoye, "Managing organizational attractiveness after a negative employer review: company response strategies and review consensus", European Journal of Work and Organizational Psychology, 2020, 1-18.

[30] Luo, Ning, Yilu Zhou, and John Shon, "Employee satisfaction and corporate performance: Mining employee reviews on Glassdoor. com", in International Conference on Information Systems, Dublin, Ireland, 2016: AIS.
[31] Chua, Alton YK, and Snehasish Banerjee, "Understanding review helpfulness as a function of reviewer reputation, review rating, and review depth", Journal of the Association for Information Science and Technology 66.2, 2015, 354-362.

[32] Hong, Y., Huang, N., Burtch, G., \& Li, C., "Culture, conformity and emotional suppression in online reviews", Journal of the Association for Information Systems, 17.11, 2016, 737-758.

[33] Lee, Hee "Andy, Rob Law, and Jamie Murphy. "Helpful reviewers in TripAdvisor, an online travel community", Journal of Travel \& Tourism Marketing 28.7, 2011, 675-688.

[34] Tausczik, Yla R., and James W. Pennebaker, "The psychological meaning of words: LIWC and computerized text analysis methods", Journal of Language and Social Psychology 29.1, 2010, 24-54.

[35] Hu, Ya-Han, and Kuanchin Chen, "Predicting hotel review helpfulness: The impact of review visibility, and interaction between hotel stars and review ratings", International Journal of Information Management 36.6, 2016, 929-944.

[36] Sionek, A., Data Jobs Listings - Glassdoor: +160k Data Related Jobs Listings Extracted from Glassdoor.

Retrieved June 23rd, 2020 from

https:/www.kaggle.com/andresionek/data-jobs-

listings-glassdoor

[37] Korfiatis, N., GarcíA-Bariocanal, E., \& SáNchezAlonso, S. (2012). Evaluating content quality and helpfulness of online product reviews: The interplay of review helpfulness vs. review content. Electronic Commerce Research and Applications, 11(3), 205-217. 